\title{
The Hydrogen-Deuterium Exchange Reaction on Cobalt Ferrite
}

\author{
R. G. SQUIRES* AND G. PARRAVANO \\ From the Department of Chemical and Metallurgical Engineering, University of Michigan, \\ Ann Arbor, Michigan
}

Received May 21, 1963

\begin{abstract}
The catalytic activity of cobalt ferrite, $\mathrm{Co}_{3-x} \mathrm{Fe}_{x} \mathrm{O}_{4}$, for the hydrogen-deuterium exchange reaction and the change in thermoelectric power of the ferrite during the adsorption of hydrogen and oxygen were investigated as functions of the catalyst composition.

The exchange reaction was carried out on catalysts with values of $x: 1.93,1.97,2.02$, and 2.07 in a flow reactor in the temperature range $60^{\circ}$ to $130^{\circ} \mathrm{C}$, at $1 \mathrm{~atm}$ pressure. It was found that the rate of the reaction was dependent upon the pretreatment of the catalyst while the activation energy increased from about 19 to $24 \mathrm{kcal} \mathrm{mole}^{-1}$ and the pre-exponential factor from $10^{30}$ to $10^{37} \mathrm{~min}^{-1}$ as the compositional parameter, $x$, progressed from 1.93 to 2.07 .

The change in thermoelectric power of compressed powder ferrite pellets during the adsorption of hydrogen and oxygen was investigated in the temperature range $88-250^{\circ} \mathrm{C}$ on two samples of cobalt ferrite, $x=1.91$ and 2.04 . At temperatures $>120^{\circ} \mathrm{C}$ hydrogen was adsorbed on the cobalt ferrite as an electron donor, and oxygen as an acceptor. However, no change in thermoelectric power was detected at lower temperatures, indicating that in the range in which the catalytic reaction was studied no net electron transfer occurred between the adsorbed molecules and the substrate.

It is concluded that the hydrogen-deuterium exchange reaction on cobalt ferrite occurred in two stages. The first is an initial activation step in which the catalytic activity increased with time. This step can be associated with the reduction of the surface of the ferrite and with the exposing of surfaec cations. In the second stage the cxchange reaction proceeded at constant activity. A reaction mechanism involving hydrogen being adsorbed dissociatively on cation-anion couples in such a way as to restore the octahedral coordination of the cations of interest $\left(\mathrm{Co}^{3+}, \mathrm{Fe}^{3+}\right)$ is in accord with the experimental results. The differences in activation energies are qualitatively related to the energies of the $\mathrm{Co}^{2+}-\mathrm{Co}^{3+}$ and $\mathrm{Fe}^{2+}-\mathrm{Fe}^{3+}$ electron transfers. A theoretically calculated reaction rate, which assumes slow hydrogen desorption, is in accord with the experimental rate.
\end{abstract}

Correlations between the chemical reactivity of metal oxides and the nature of the electronic defects of the solid have been generally deduced from a comparison of the catalytic behavior of chemically different solids. In this manner the possibility arises that the reported relationships may be more dependent upon variables controlling the gross chemical nature of the solid than upon the sign of the majority carriers. This possi-

* National Science Foundation Fellow, 1958-61* Present Address: School of Chemical Engineering, Purdue University, West Lafayette, Indiana. bility is strengthened by recent investigations on the catalytic activity of $p$ - and $n$-type germanium samples for the hydrogen-deuterium reaction (1). These studies showed that the catalytic activity was not appreciably influenced by the sign of the majority of the electrical carriers in germanium. Furthermore, it has not been possible in most instances to convincingly differentiate between the role of electronic and ionic defects (2) on surface reactivity. Thus, a correlation between electrical properties and catalytic activity cannot, in 
general, be inferred whenever variations in both of these properties are obtained by modifying different variables of the system. In a similar fashion, the extensive work carried out to derive a relationship between the electronic and catalytic effects of doping in semiconducting catalysts has produced a situation clouded by many uncertainties. At present, it is not possible to predict whether marked qualitative differences in the catalytic activity of $n$ - against $p$-type semiconductors or among different types of doping are to be expected. Similarly, no predictions can be made on the catalytic behavior of substances with mixed $n$ and $p$-type conductivities (double carrier conductors).

Some of these ambiguities may be clarified by studying the catalytic activity of solid compounds with a well-defined chemical composition (ion defects) and electronic behavior (electron defects) and which permit modification of the latter without appreciably influencing the former. Furthermore, it is advantageous to use compounds with which the investigation of the whole range of $p$ - and $n$-type properties is feasible without drastically modifying the chemical composition of the solid. These requirements are met by some oxide compounds with a spinel structure. In these binary systems, the valences of the metal ions are determined by the tendency to form an ideal spinel structure with the ratio of 3 to 4 of metal to oxygen ions. As a result, these solids do not contain an appreciable number of lattice vacancies, thus justifying the assumption of a fixed metal/oxygen ratio during adsorption and catalysis. Since the ratio may influence the catalytic activity (B), this situation permits a closer investigation of the effect of adsorption and catalysis on the electronic equilibrium of the system.

In cobalt ferrite, $\mathrm{Co}_{3-x} \mathrm{Fe}_{x} \mathrm{O}_{4}$, the stoichiometric composition, $\mathrm{CoFe}_{2} \mathrm{O}_{4}$, contains all of the cobalt ions as $\mathrm{Co}^{2+}$ and all of the iron ions as $\mathrm{Fe}^{3+}$. Thus $x=2$. For $x>2$ the compound is an $n$-type semiconductor and the excess iron is incorporated as $\mathrm{Fe}^{2+}$. For $x<2$, the ferrite contains excess cobalt which is incorporated as $\mathrm{Co}^{3+}$. Variations in $x$ of 0.05 per molecule for values of $x \approx 2$ are sufficient to cause drastic changes in electrical conductivity ( 7 orders of magnitude) and thermoelectric power $(+800$ to $-600 \mu$ volt $\left.{ }^{\circ} \mathrm{C}^{-1}\right)$ (4). Thus $p$ - and $n$-type samples can be prepared having essentially similar chemical compositions and with the concentration of excess electrons and electron holes simply controlled by the concentration of $\mathrm{Fe}^{2+}$ and $\mathrm{Co}^{3+}$, respectively. These effects can be achieved without the necessity of introducing foreign ions into the spinel phase. This eliminates most of the difficulties inherent in the method of doped semiconductors (chemical and electronic effects of the additions, their distribution throughout the solid phase and surfacc) and it allows a more direct test of the role of the sign of the majority carriers or of the presence of double carrier conduction on catalytic reactions. Finally, this class of spinel compounds enables verification of the claim that only trivalent cations in spinel structures represent sites of catalytic activity (5).

In order to analyze some of these considerations, we have performed experiments on the rate of the isotopic exchange reaction between hydrogen and deuterium on cobalt ferrite samples, with different $\mathrm{Fe} / \mathrm{Co}$ ratios. The results of this study are summarized in the present communication, together with observations on the effect of hydrogen and oxygen adsorption on the thermoelectric power of cobalt ferrite.

\section{EXPERIMENTAL \\ Materials}

Weighed amounts of reagent grade cobalt carbonate (J. T. Baker) and iron oxide (Allied Chemical and Dye) were mixed and wet ball-milled in acetone for $4 \mathrm{hr}$. After drying overnight, the mixture was fired at $1050^{\circ} \mathrm{C}$ for $4 \mathrm{hr}$ in air. The material was then crushed, screened, and compressed in a $3 / 8$ inch diameter mold. The pellets were further fired for $10 \mathrm{hr}$ at $1350^{\circ} \mathrm{C}$ in air, air-quenched, crushed, screened, and the fraction between 40 and 100 mesh was used as the catalyst in the hydrogen-deuterium exchange runs. The BET surface area of these samples, determined by low-temperature nitrogen adsorption, was found to be $0.08 \mathrm{~m}^{2} \mathrm{~g}^{-1}$. This preparation resulted in cobalt ferrite samples which, due to their low surface to 
volume ratio, showed no measurable variation in thermoelectric power or electrical resistivity during the adsorption of hydrogen or oxygen after $48 \mathrm{hr}$ of contact with the gas atmosphere at $200^{\circ} \mathrm{C}$. It was, therefore, necessary for investigations on the effect of gas adsorption on thermoelectric power to prepare samples with higher surface area. For this purpose, the material from the $1050^{\circ} \mathrm{C}$ firing was mixed on rolling mill for $17 \mathrm{hr}$ and fired at $1100^{\circ} \mathrm{C}$ for $8 \mathrm{hr}$ in air, airquenched, crushed, and screened. The fraction between 40 and 100 mesh was compressed at 20,000 psi into $3 / 8$ inch diameter pellets, which were used in the thermoelectric power studies. The BET surface area of these samples was found to be equal to $2.0 \mathrm{~m}^{2} \mathrm{~g}^{-1}$.

Spinel samples were made having four different $\mathrm{Fe} / \mathrm{Co}$ ratios, two of which resulted in $n$-type and two in $p$-type ferrite. The ratios were determined by comparing the catalyst samples with three samples of known $\mathrm{Fe} / \mathrm{Co}$ ratios by means of X-ray fluorescent spectrometry. The standard samples were made from the same starting materials, ball-milled, and pressed into 1-inch pellets. X-ray fluorescence analysis was carried out 12 times on each side of every catalyst pellet. The homogeneity of the samples was checked by grinding the surface and exposing a new layer of material between each analysis. The $\mathrm{Fe} / \mathrm{Co}$ ratio was also determined by wet chemical analysis.
Particle size distribution of the ferrite powder was determined from electronmicrographs at $11000 \times$ magnification.

Prepurified hydrogen (Matheson Co.) and deuterium (General Dynamics Corp.) were obtained from commercial tanks and further purificd by passage through a "Deoxo" catalytic purifier, followed by a phosphorous pentoxide drying column. Helium from a commercial tank was purified by passage through hot copper oxide and an activated charcoal cold trap. Oxygen (Matheson Co.) was purified by passage through the activated charcoal cold trap.

\section{Apparatus}

The rate of the hydrogen-deuterium exchange reaction was studied in a flow system at atmospheric pressure. A stream of hydrogen containing $2 \%$ deuterium flowed at constant rate from a glass-lined, pressurized mixing tank through a preheater and the catalyst bed kept at constant temperature $\left( \pm 0.1^{\circ} \mathrm{C}\right)$ in a Pyrex reactor. After leaving the reactor, the exit stream was sampled and the sample analyzed by a mass spectrometer.

The thermoelectric power of the ferrite pellets was measured in a cell whose details are shown in Fig. 1. A modified Dresser coupling was used to support the sample holder and Pyrex cover. The sample pellet was mounted between two 20 -mil platinum discs, supported by $2-\mathrm{mm}$ bore capillary

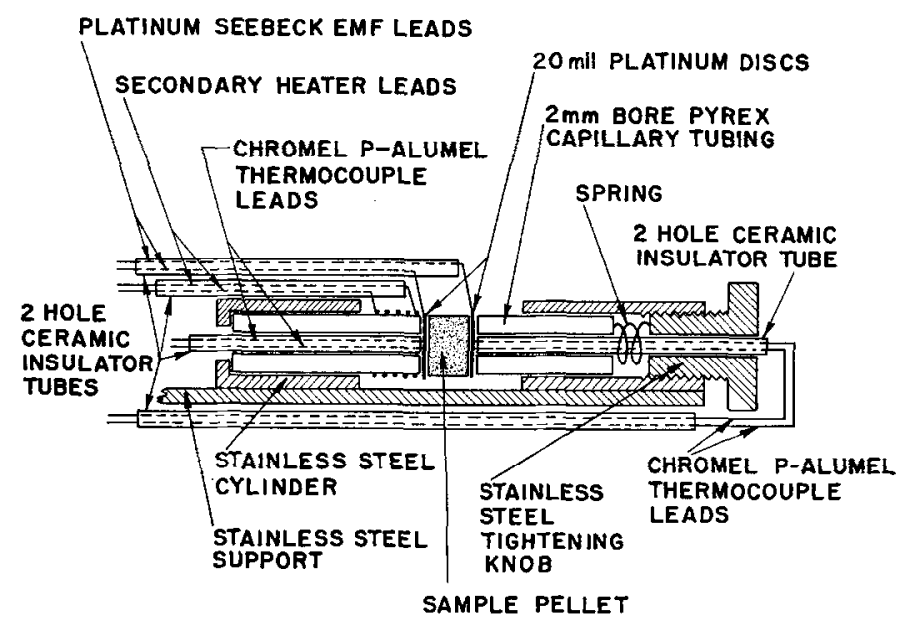

FIG. 1. Thermoelectric power apparatus. 
Pyrex tubing. The capillary tubes were mounted inside hollow stainless steel cylinders which were welded to the sample holder support. The sample was tightened in place by means of a knob, threaded into the outer cylinder. Temperature gradients across the sample were obtained by means of an auxiliary heating coil wound on the inner glass capillary. Chromel-Alumel thermocouples and $\mathrm{Pt}$ wire (for emf measurements) were welded on each platinum disc on the side opposite the sample. The wires were led outside the cell through a Kovar seal. Temperatures were measured by means of a K-3 Leeds and Northrup potentiometer and an electronic DC null detector. Thermal emf's were determined by means of the K-3 potentiometer and a DC microvolt ammeter, used as a high impedance null detector.

\section{Procedure}

In the course of preliminary runs, it was found that the rate of the exchange reaction could be conveniently studied in the temperature range $110^{\circ} \mathrm{C}$ and upwards, after having subjected the catalyst to a treatment with hydrogen at $200^{\circ} \mathrm{C}$ for $8 \mathrm{hr}$. However, following this procedure the activity of the catalyst remained constant only for the duration of the kinetic investigations with two different gas flow rates (high-temperature runs). Subsequently it was established that reproducible catalytic activity for long periods of time, at all flow rates studied, could be achieved upon submitting the catalyst to a hydrogen pretreatment lasting $12 \mathrm{hr}$ at $200^{\circ} \mathrm{C}$ (low-temperature runs). The constancy of the activity was often checked by repeating runs after having completed the usual sequence of experiments at different fow rates, for periods of time of continuous operation of $6 \mathrm{hr}$. The activated samples could be rapidly deactivated if exposed to air or oxygen. The per cent conversion, $p$, was determined from observations at at least three different flow rates (from 10 to $35 \mathrm{~cm}^{3} \mathrm{~min}^{-1}$ ) at the same temperature. Activation energies and preexponential factors were computed from the cquation*:

* For explanation of the symbols see the section at the end of the paper. $k t=\ln \left(\frac{1}{1-p}\right)=\frac{k_{0} \varphi T_{\mathrm{a}} P}{v_{\mathrm{a}} T P_{\mathrm{a}}}\left(\exp -\frac{E}{R T}\right)$

Equation (1) assumes first order kinetics and the applicability of the Arrhenius equation. Plots of $\log \left(v_{\mathrm{a}} T\right)$ vs. $(1 / T)$ at constant $p$ and of $\log [1 /(1-p)]$ vs. $1 / v_{\mathrm{a}}$ at constant $T$ were used to calculate the activation energy and pre-exponential factors, respectively.

To minimize electrical contact resistance in the determination of the thermoelectric power, the ends of the ferrite pellet were coated with graphite. The pellet was clamped between the platinum dises and the Pyrex envelope put in place. The cell was evacuated to $10^{-4} \mathrm{~mm} \mathrm{Hg}$, subsequently filled with purified helium and evacuated again to $10^{-1} \mathrm{~mm} \mathrm{Hg}$. This cycle was repeated five times. The cell was finally filled with helium at atmospheric pressure and a temperature difference of about $3^{\circ} \mathrm{C}$ was set across the pellet. Helium pressure was then reduced to $30 \mathrm{~cm} \mathrm{Hg}$ and 2 to $6 \mathrm{~cm} \mathrm{Hg}$ of hydrogen (or oxygen) gas was introduced. The pressure was increased to $76 \mathrm{~cm} \mathrm{Hg}$ with prepurified helium. Measurements of the thermal emf were taken and continued until steady state was reached. Additional details on the equipment used, preparation of samples, and analytical procedures can be found elsewhere $(6)$.

\section{RESULTS}

\section{Catalyst Characterization}

$\mathrm{X}$-ray diffraction patterns and microscopic examination of all the compositions prepared indicated that the catalyst was a single phase spinel $(4,7)$. Particle size of the catalyst samples was between 0.15 to 0.5 $\mathrm{mm}$, and the picnometric density was $5.2 \mathrm{~g} \mathrm{~cm}^{-3}$.

Figure 2 presents an electronmicrograph of the spinel used in the thermoelectric power experiments. Particle-size distribution of these samples showed a normal distribution when plotted on a logarithmic scale (Fig. 3) which gives a skewed curve when plotted on a linear scale. The skewed distribution, found also in previous work $(8)$, is probably due to sintering of the 


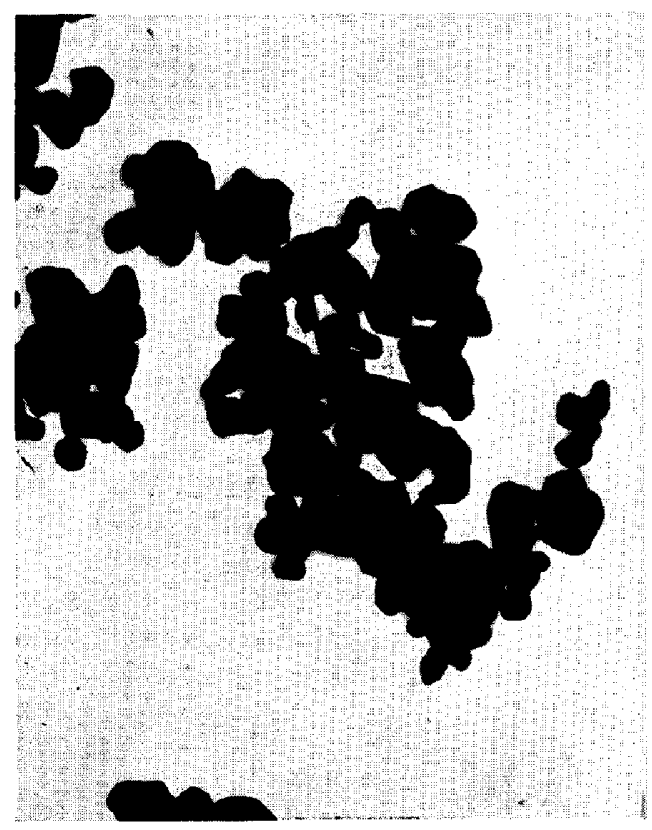

FIG. 2. Electron photomicrograph of separated ferrite particles. $(13,330 \times)$.

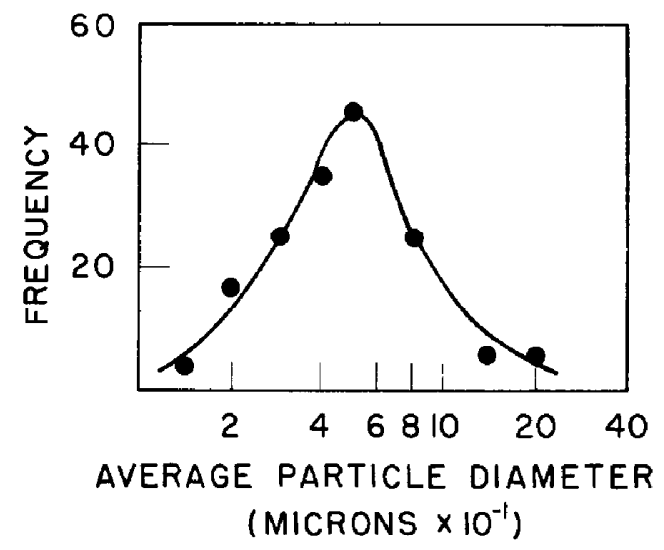

FIG. 3. Particle size distribution for separated ferrite particles.

ferrite powder. Assuming spherical shape, the average particle size of the spinel samples used in the thermoelectric experiments can be calculated to be $0.56 \mu$, in agreement with the value determined electron-microscopically (Fig. 3). For electrical characterization of the samples thermoelectric power measurements were used. The values of the thermoelectric power of the spinel compositions determined in air are reported in Fig. 4, together with the results from previous investigations (4).

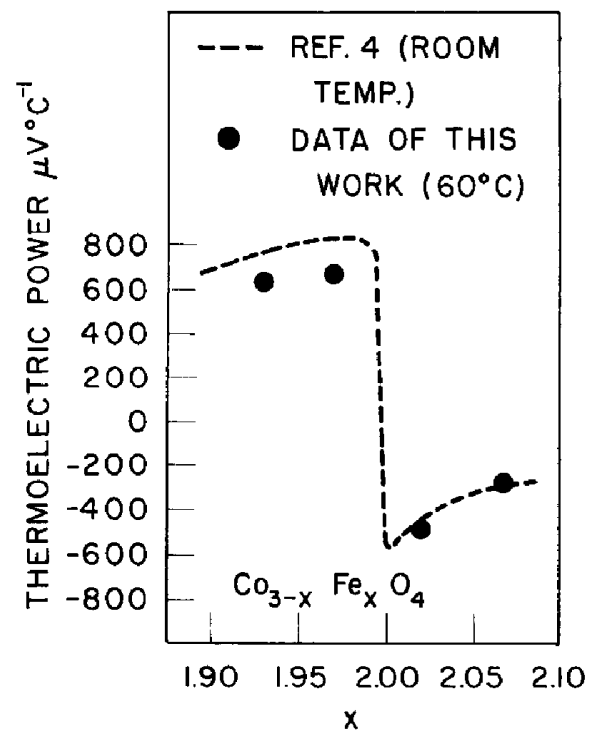

FIG. 4. Thermoelectric power of cobalt ferrite as a function of composition. Ambient: air.

\section{Thermoelectric Power Measurements}

In preliminary runs, no variation in thermoelectric power of the sample was found with varying $\Delta T$ with $\Delta T$ up to $25^{\circ} \mathrm{C}$. Generally, a value of $\Delta T \approx 3^{\circ} \mathrm{C}$ was used. At $\Delta T \cong 0$, a small thermal emf was observed (about 20 [ $\mu$ volt]), its value being approximately constant in helium, oxygen, or hydrogen atmospheres. Typical plots showing the change in thermoelectric power during gas adsorption are given in Figs. 5, 6,7 , and 8 . A summary of the results of all of the thermoelectric runs is given in Table 1.

\section{Hydrogen-Deuterium Exchange Reaction}

Typical results on the effect of gas flow rate on the conversion at constant temperature and of temperature at constant flow rate are reported in Figs. 9 and 10. A typical run is shown in Fig. 11, while a summary of the computed activation energies and preexponential factors is given in Table 2.

Rate experiments were also performed on mechanically mixed samples, with compositions at opposite sides of $x=2$, with or without sintering. These results are presented in Table 3. 


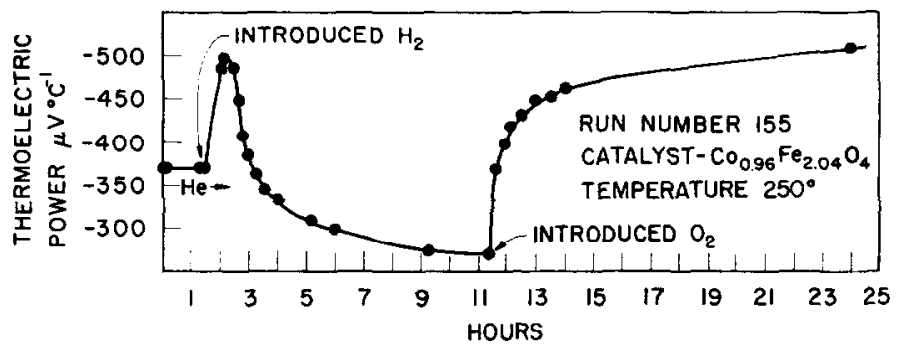

FIG. 5. Variation of thermoelectric power with time in helium, hydrogen, and oxygen atmospheres; temperature $=250^{\circ} \mathrm{C}$.

TABLE 1

Thermoelectric Power Change During the Adsorption of Hydrogen and Oxygen on Cobalt Ferrite

\begin{tabular}{|c|c|c|c|c|}
\hline \multirow[b]{2}{*}{$\begin{array}{l}\text { Run } \\
\text { no. }\end{array}$} & \multicolumn{3}{|c|}{ Gas adsorbed } & \multirow[b]{2}{*}{$\begin{array}{c}\text { Temperature } \\
\left({ }^{\circ} \mathrm{C}\right)\end{array}$} \\
\hline & $x$ & Oxygen ${ }^{a}$ & Hydrugen ${ }^{a}$ & \\
\hline 112 & 1.91 & (1) $\hat{i}$ & - & 250 \\
\hline 113 & 1.91 & - & (1) $\downarrow$ & 250 \\
\hline 114 & 2.04 & $(2) \downarrow$ & $(1) \uparrow$ & 250 \\
\hline 119 & 2.04 & (2) $\downarrow$ & $(1) \uparrow$ & 250 \\
\hline 120 & 1.91 & (2) $\uparrow$ & (1) $\downarrow$ & 250 \\
\hline 126 & 1.91 & (1) $\uparrow$ & $(2) \downarrow$ & 250 \\
\hline 138 & 2.04 & (2) & (1) \ & 250 \\
\hline 139 & 2.04 & & $(1) \searrow$ & 250 \\
\hline 141 & 1.91 & (1) $\uparrow$ & (2) $\downarrow$ & 250 \\
\hline 142 & 2.04 & (2) $\uparrow$ & (1) \ & 250 \\
\hline 143 & 1.91 & $(2) \uparrow$ & (1) $\downarrow$ & 250 \\
\hline 147 & 1.91 & (2) $\uparrow$ & (1) $\downarrow$ & 250 \\
\hline 149 & 1.91 & (2) $\nearrow$ & $(1) \rightarrow$ & 140 \\
\hline 150 & 2.04 & $(2) \rightarrow$ & $(1) \rightarrow$ & 88 \\
\hline 152 & 1.91 & (1) $\uparrow$ & (2) $\downarrow$ & 250 \\
\hline 153 & 1.91 & (1) $\uparrow$ & (2) $\downarrow$ & 250 \\
\hline 155 & 2.04 & $(2) \uparrow$ & $(1) \searrow$ & 250 \\
\hline $155^{a}$ & 2.04 & $(1)-\nearrow+b$ & $(2)+\searrow-b$ & 250 \\
\hline 157 & 2.04 & 一 & (1) $\downarrow$ & 160 \\
\hline 158 & 2.04 & - & $(1) \rightarrow$ & 88 \\
\hline $158^{a}$ & 2.04 & - & $(1) \rightarrow$ & 120 \\
\hline 159 & 2.04 & 一 & (1) $\downarrow$ & 180 \\
\hline
\end{tabular}

$a$ The arrows show the direction of change of the absolute value of thermoelectric power upon admittance of the gas. The numbers (1), (2) refer to the order in which the gases were admitted, i.e., compare Runs 155, 157, 150, and 155a with Figures $5,6,7$, and 8 .

${ }^{b}$ In Run 155a the thermoelectric power changes from - to + as oxygen was first introduced and from + to - when hydrogen was introduced. The + and - signs on the arrows indicate this change.

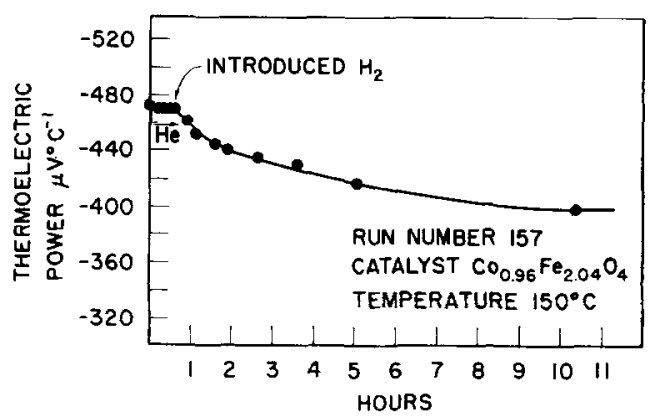

Fic. 6. Variation of thermoelectric power with time in helium, and hydrogen atmospheres; temperature $=150^{\circ} \mathrm{C}$.

\section{Discussion}

\section{Thermoelectric Power Results}

The results and conclusions on the effect of the chemisorption of hydrogen and oxygen on the thermoelectric power of the ferrite catalysts can be summarized as follows:

1. No variation in the thermoelectric power of the catalyst was detected whenever a surrounding atmosphere of helium was replaced by hydrogen at reaction conditions $\left(60^{\circ}\right.$ to $\left.130^{\circ} \mathrm{C}\right)$.

2. At higher temperatures (up to $250^{\circ} \mathrm{C}$ ) variations in the thermoelectric power of the ferrite upon hydrogen or oxygen adsorption were dependent upon the type of semiconductivity displayed by the samples (single or double carrier semiconductor), hydrogen and oxygen adsorptions entailing in both cases electron donation to or acceptance from the solid, respectively.

These results can be interpreted on the 


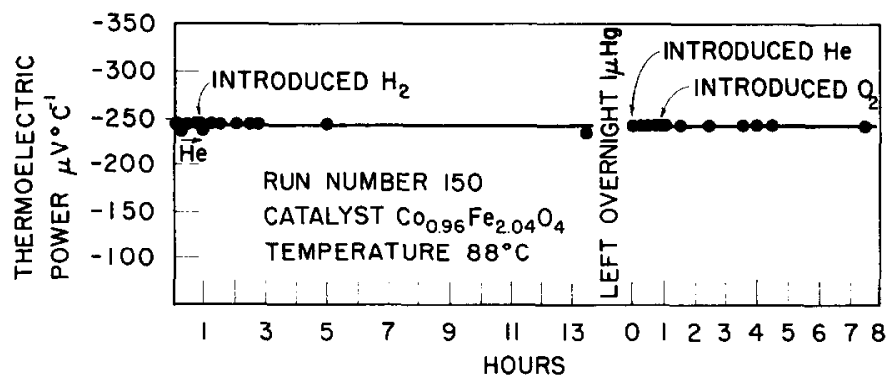

FIG. 7. Variation of thermoelectric power with time in helium, hydrogen, and oxygen atmospheres; temperature $=88^{\circ} \mathrm{C}$.

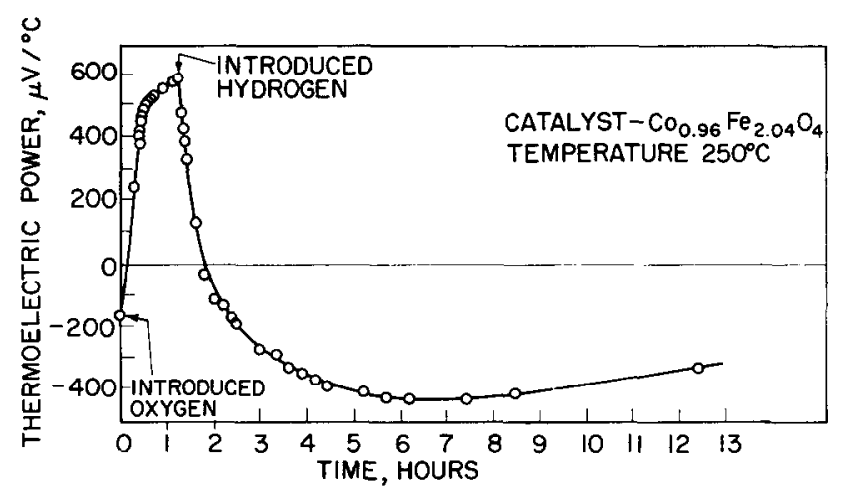

Fig. 8. Run number 155a; variation of thermoelectric power with time in oxygen and hydrogen atmospheres.

TABLE 2

Summary of Activation Energy and Pre-exponentill Factors for Hydrogen-Deuterium Exchange Experiments on Cobalt Ferrite

\begin{tabular}{|c|c|c|c|c|}
\hline \multirow[b]{2}{*}{$x$} & \multicolumn{2}{|c|}{ High-temperature runs } & \multicolumn{2}{|c|}{ Low-temperature runs } \\
\hline & $\begin{array}{c}\text { Act, } \\
\text { energy } \\
(\text { kcal mole } \\
-1)\end{array}$ & $\begin{array}{l}\text { Pre-exp } \\
\text { factor } \\
\ln \left(k_{0}\right)\end{array}$ & $\begin{array}{c}\text { Act. } \\
\text { energy } \\
\left(\text { kcal mole }^{-1}\right)\end{array}$ & $\begin{array}{l}\text { Pre-exp } \\
\text { factor } \\
\ln \left(k_{0}\right)\end{array}$ \\
\hline \multirow[t]{3}{*}{1.93} & 19.4 & 29.2 & 18.3 & 30.1 \\
\hline & 19.9 & 30.2 & 18.5 & 30.2 \\
\hline & 18.4 & 27.6 & - & 一 \\
\hline \multirow[t]{3}{*}{1.97} & 18.7 & 27.5 & 19.0 & 30.8 \\
\hline & 18.5 & 26.9 & 18.6 & 30.1 \\
\hline & 18.8 & 27.2 & - & - \\
\hline \multirow{4}{*}{2.02} & 23.2 & 33.4 & 23.9 & 37.8 \\
\hline & 23.0 & 33.0 & 23.1 & 36.1 \\
\hline & 22.9 & 33.0 & - & - \\
\hline & 22.3 & 32.0 & - & 一 \\
\hline \multirow[t]{2}{*}{2,07} & 23.3 & 33.8 & 23.4 & 37.3 \\
\hline & 22.4 & 32.1 & 23.9 & 37.9 \\
\hline
\end{tabular}

basis of the known electronic model of the spinel (4), and can be conveniently discussed according to three temperature ranges. At high temperatures $\left(180-250^{\circ} \mathrm{C}\right)$
$\mathrm{Co}^{3+}$ and $\mathrm{Fe}^{2+}$ ions, which are located at $0.06 \mathrm{ev}$ above the valence band or below the conduction band, respectively, are completely ionized and electrons are thermally 


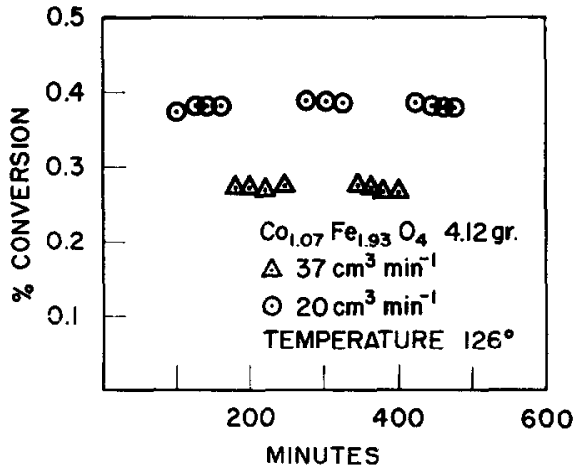

Fig. 9. The effect of changes in flow rate on per cent conversion for the hydrogen-deuterium reaction at constant temperature.

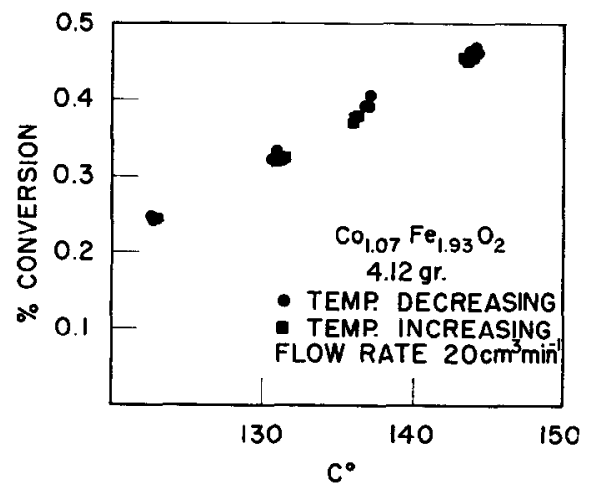

FIG. 10. Per cent conversion as a function of temperature at constant flow rate.

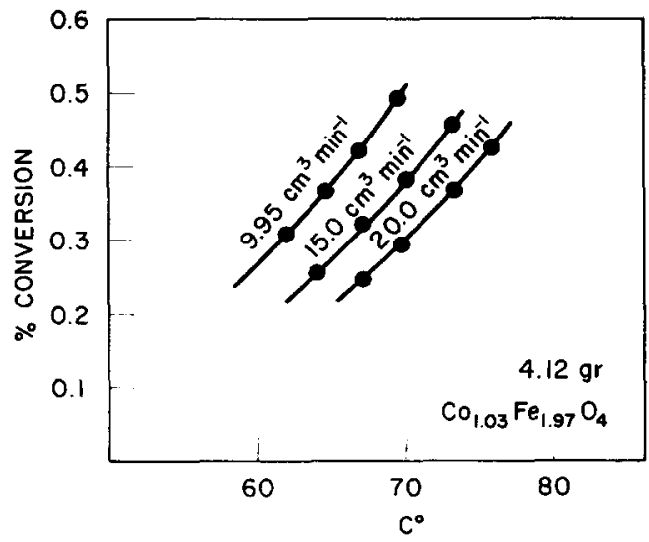

FIG. 11. A typical hydrogen-deuterium exchange run.

excited from the valence band into the conduction band (gap width $0.55 \mathrm{ev}$ ). As a result, cobalt ferrite becomes a two carrier
TABLE 3

Activation Energies and Pre-exponential Factors for Runs Using Mixed Sintered Catalysts

\begin{tabular}{|c|c|c|c|}
\hline Sample & $\begin{array}{c}\text { Time and } \\
\text { temperature } \\
\text { of } \\
\text { sintering }\end{array}$ & $\begin{array}{c}\text { Act. } \\
\text { energy } \\
(\text { kcal mole-1) }\end{array}$ & $\begin{array}{l}\text { Pre-exp } \\
\text { factor } \\
\ln \left(k_{0}\right) \\
\left(\min ^{-1}\right)\end{array}$ \\
\hline \multirow{5}{*}{$\left.\begin{array}{l}50 \% \mathrm{Co}_{1.07} \mathrm{Fe}_{1 . .8} \mathrm{O}_{4} \\
50 \% \mathrm{Co}_{0.83} \mathrm{Fe}_{2.07} \mathrm{O}_{4}\end{array}\right\}$} & 0 & 20.7 & 33.5 \\
\hline & $1 \mathrm{hr}$ & 21.6 & 34.2 \\
\hline & $850^{\circ} \mathrm{C}$ & & \\
\hline & $\begin{array}{l}1 \mathrm{hr} \\
990^{\circ} \mathrm{C}\end{array}$ & 21.1 & 34.2 \\
\hline & $\begin{array}{l}1 \mathrm{hr} \\
1120^{\circ} \mathrm{C}\end{array}$ & 22.1 & 34.3 \\
\hline
\end{tabular}

semiconductor with electrons in the conduction band contributing to the $n$-type conductivity and the holes in the valence band contributing to the $p$-type conductivity. In this temperature range thermoelectric power is given by $(4)$ :

$$
Q T=\frac{-n_{1} \mu_{1}\left(E_{\mathrm{g}}-E_{\mathrm{f}}+\alpha\right)+n_{2} \mu_{2}\left(E_{\mathrm{f}}+\beta\right)}{n_{1} \mu_{1} e+n_{2} \mu_{2} e}
$$

where $E_{\mathrm{f}}$ and $\left(E_{\mathrm{g}}-E_{\mathrm{f}}\right)$ are logarithmic functions of carrier concentration. For $n_{1} \gg n_{2}$ (or $n_{2} \gg n_{1}$ ), Eq. (2) reduces to:

$$
\begin{array}{ll}
e Q T=-\alpha-k T \ln \left(N / n_{1}\right) & \left(n_{1} \gg n_{2}\right) \\
e Q T=+\beta+k T \ln \left(N / n_{2}\right) & \left(n_{2} \gg n_{1}\right)
\end{array}
$$

In air cobalt fermite becomes a two carrier semiconductor at temperatures above approximately $160^{\circ} \mathrm{C}$ (4).

Let us consider samples with $x>2$, and assume that hydrogen adsorption produces energy levels close to the donor levels already present in the spinel. At $250^{\circ} \mathrm{C}$ the former will be completely ionized and the electrons released by hydrogen adsorption should increase the value of the n-type thermoelectric power, as predicted by Eq. (2). When the increase in free electron population reaches the condition $n_{1} \gg n_{2}$, the magnitude of $n$-type thermoelectric power should begin to decrease according to Eq. (3). If oxygen is now admitted to the surface, electrons will be trapped by adsorbed oxygen and the value of the thermoelectric power should again increase. Oppo- 
site effects should occur if the order of admission of hydrogen and oxygen is exchanged. These facts are clearly demonstrated in runs $138,139,142,155$, and $155 \mathrm{a}$ (Table 1). In run 155a (Fig. 8), oxygen was adsorbed at $250^{\circ} \mathrm{C}$ on an $n$-type ferrite. As chemisorption proceeded the condition $n_{1}<n_{2}$ was reached and the thermoelectric power changed from an initial value of -170 to a value of +600 [ $\mu$ volt $\left.{ }^{\circ} \mathrm{C}^{-1}\right]$, where it began to level off as $n_{1}$ became much less than $n_{2}$. Upon contacting this surface with hydrogen, the thermoelectric power switched from +600 to $-420\left[\mu\right.$ volt $\left.{ }^{\circ} \mathrm{C}^{-1}\right]$. At this point, $n_{1} \gg n_{2}$ and as predicted by Eq. (3) the value of thermoelectric power began to decrease as $n_{1}$ increased further.

The order of magnitude of the amounts of hydrogen and oxygen needed for the transition from two carrier to the single carrier semiconductivity may be estimated by means of the known electronic structure of the ferrite (/). Assuming that the maximum value of the thermoelectric power in our experiments is solely controlled by the adsorbed species, it can be calculated that a surface coverage of about $2 \%$ is necessary to change the thermoelectric power from the negative minimum to the positive maximum under the conditions of Fig. 8.

At temperatures between $100^{\circ}$ and $200^{\circ} \mathrm{C}$, the acceptor (or donor) species are thermally ionized but intrinsic conduction is negligible, and Eqs. (3) or (4) apply. In this case the value of the thermoelectric power of an $n$-type sample should be decreased by hydrogen and increased by oxygen adsorption. In a similar manner, but with opposite results, the magnitude of the thermoelectric power of the $p$-type ferrite should vary. These effects are demonstrated in Table 1 . The suggested interpretation assumes that the kinetic terms $\alpha$ and $\beta$ in Eqs. (2), (3), and (4) are not appreciably affected by hydrogen or oxygen adsorption.

At temperatures between $80^{\circ}$ and $120^{\circ} \mathrm{C}$, cobalt ferrite becomes a single carrier semiconductor with no intrinsic conduction. Therefore, the effect of the transfer of electrons from adsorbed hydrogen or oxygen is expected to be similar to that observed in the intermediate temperature range (100$200^{\circ} \mathrm{C}$ ). However, the results presented in the previous section show that no change in thermoelectric power was detected during the adsorption of hydrogen or oxygen. This fact points to chemisorption processes entailing no appreciable electron transfer between the adsorbed molecules and the catalyst surface. This is not an unexpected conclusion. On zinc oxide below $100^{\circ} \mathrm{C}$ there are presently recognized four differcnt types of hydrogen chemisorptions, none of which influences the electrical conductivity of the substrate (9). Similar results are known for the chemisorption of hydrogen on transition metal oxides, having an electron configuration close to that of cobalt ferrite (10).

\section{Hydrogen-Deuterium Exchange}

The significant experimental results on the catalytic activity of cobalt ferrite may be summarized as follows:

1. After a suitable activation, cobalt ferrite was found to catalyze the isotopic exchange reaction between hydrogen and deuterium at temperatures from $60^{\circ} \mathrm{C}$ upwards; lower reaction temperatures could probably be obtained with a more extensive activation procedure.

2 . The rate of reaction was dependent upon the condition of catalyst activation.

3 . The activation energy of the reaction varied from 18.3 to $23.9 \mathrm{kcal}$ mole ${ }^{-1}$, when $x$ was altered from 1.93 to 2.07 ( $\mathrm{Fe} / \mathrm{Co}$ molar ratios of 1.80 and 2.22 , respectively).

4. A mechanical mixture of two compositions at opposite sides of $x=2$, with or without previous sintering, produced a catalyst of activity intermediate between those of the original compositions.

The correlation of the experimental results on the basis of the numerical values of the rate of the exchange reaction is not possible because of the critical dependence of the former upon the conditions of previous treatment of the catalyst. Thus, by varying the time of hydrogen treatment at $200^{\circ} \mathrm{C}$ from 8 to $12 \mathrm{hr}$, the reaction conversion on $\mathrm{Co}_{1.07} \mathrm{Fe}_{1.93} \mathrm{O}_{4}$ at $75^{\circ} \mathrm{C}$ and constant flow rate of $20 \mathrm{~cm}^{3} \mathrm{~min}^{-1}$ increased by a factor of about 15. This effect is common to most metal oxides. Previous discussions on reactivity patterns of metal oxides for the hydrogen-deuterium exchange reaction, based solely on the rate of reaction, have produced 
conflicting views because of the rather poor reproducibility of the values of the reaction rate (11). To a large extent, the deduced patterns are dependent upon the actual pretreatment procedure. Activation energies are generally not influenced much by the catalyst pretreatment. In the present in. vestigation, chemically similar catalysts, whose activity was modified by about an order of magnitude by the pretreatment procedure did possess about the same value of the activation energy (Table 2). Because of this effect, energies of activation were used in the present study as primary sources for the discussion of the experimental rcsults, although the interpretation of the former cannot be definitive since the mechanism of the exchange reaction at metal oxide surfaces is not yet fully established.

In stoichiometric cobalt ferrite, all $\mathrm{Co}^{2+}$ ions and one-half of the $\mathrm{Fe}^{3+}$ ions occupy octahedral sites in the spinel structure, while the remaining one-half of the $\mathrm{Fe}^{3+}$ ions are distributed in the tetrahedral positions (inverse spinel structure). For samples with $x \neq 2, \mathrm{Co}^{3+}$ and $\mathrm{Fe}^{2+}$, which have a similar $3 d^{6}$ electron configuration, prefer octahedral sites. Thus, the latter type of sites have the possibility of accomodating in a simple fashion cobalt and iron ions of +2 and +3 electrical charge. Since a similar situation cannot easily be brought about in the tetrahedral sites, we shall discuss the reported catalytic effects of samples with different values of $x$ in relation to those surface positions which partake in a sixfold coordination group.

In order to visualize the relation of surface octahedral cations to surrounding ions and the various possibilities of adsorption sites on cobalt ferrite, it is instructive to briefly consider the structure of some typical crystal planes of the spinel phase. The (100), (110), and (i10) planes are schematically represented in Fig. 12. These planes are likely to occur on spinel surfaces since they fulfill the condition of high atomic density and relatively low number of chemical bonds between them. Some characteristics of these planes are listed in Appendix I. The surface configurations of interest in relation to adsorption and catalysis are likely to be controlled by the tendency to restore at the surface the sixfold coordination of the cations. Thus, if one considers cations as primary adsorption sites, an inspection of Fig. 12 shows different types of possible adsorption centers: in the (100) planes one adatom for every distorted, square pyramid, surface complex, $\left(\mathrm{Me}^{3+}\right)\left(\mathrm{O}^{2-}\right)_{5}$, is sufficient to restore octahedral coordination around the cation, while in the (i10) plane two adatoms are needed to achieve the same final state. Furthermore, on the (100) plane $\mathrm{Fe}^{2+}, \mathrm{Fe}^{3+}, \mathrm{Co}^{2+}, \mathrm{Co}^{3+}$ can be present, while the (110) plane contains $\mathrm{Co}^{2+}, \mathrm{Co}^{3+}$ ions only. Several types of adsorption complexes are conceivable on these surfaces and some possibilities have already been discussed (12).

In the original surface covered with oxygen (Fig. 13a), each surface cation is probably fully coordinated. The high-temperature treatment of this surface with hydrogen is a forerunner of surface reduction with elimination of water. This course has been extensively established for several metal oxides, including those of transition metals. Let us consider samples with $x<2$. The surface octahedral complexes, $\mathrm{Co}^{3+}\left(\mathrm{O}^{2-}\right)_{6}$, will be reduced to a lower coordination (square pyramid, tetrahedral) by loss of oxygen (Fig. 13b, c):

$$
\begin{aligned}
\mathrm{Co}^{3+}\left(\mathrm{O}^{2-}\right)_{6}+\mathrm{H}_{2} \rightarrow & \mathrm{Co}^{3+}\left(\mathrm{O}^{2-}\right)_{4}\left(\mathrm{OH}^{-}\right)_{2}+2 \mathrm{e} \\
\searrow & \mathrm{Co}^{3+}\left(\mathrm{O}^{2-}\right)_{5}+\mathrm{H}_{2} \mathrm{O}+2 \mathrm{e}
\end{aligned}
$$

Reaction (5) involves electron transfer to the substrate, in accordance with the hightemperature results of the thermoelectric power measurements, and it brings about a reduction in the number of surface anions. This interpretation of the catalyst activation step is consistent with the known fact that catalytic activation of metal oxides for the hydrogen-deuterium reaction can also be achieved by vacuum pretreatment, without the presence of hydrogen (14). The resulting lower coordination of surface cations leaves the latter more exposed and the initial surface complexes more distorted and disrupted.

Room-temperature hydrogen adsorption on such a surface can easily occur by heterolytic fission (12) which restores some of the original coordination of the cations (Fig. 13d) : 


$$
\begin{aligned}
& \mathrm{Co}^{3+}\left(\mathrm{O}^{2-}\right)_{5}+\mathrm{H}_{2} \rightarrow \mathrm{Co}^{2+}(\mathrm{H})\left(\mathrm{OH}^{-}\right)\left(\mathrm{O}^{2-}\right)_{4} \text { or } \\
& \mathrm{Co}^{3+}\left(\mathrm{O}^{2-}\right)_{5}+\mathrm{H}_{2} \rightarrow \mathrm{Co}^{3+}\left(\mathrm{O}^{2-}\right)_{4}\left(\mathrm{H}^{-}\right)\left(\mathrm{OH}^{-}\right)
\end{aligned}
$$

The first adsorption reaction of hydrogen will not result in an electron transfer involving the metal ion if the $\mathrm{Co}^{3+}$ ions are considered fast trapping centers for electrons, while in the second reaction no change in the oxidation state of the metal ion is required and therefore no effect on the electron population of the solid will result. This is consistent with the results reported in the previous section on the effect of hydrogen on the thermoelectric power at temperatures approximately $<100^{\circ} \mathrm{C}$ (Fig. 7). Upon such a hydride surface, hydrogen exchange can occur by desorption from adjacent groupings (12) (Fig. 13e). For samples with $x>2$, it is possible to consider a similar activation and reaction scheme occurring on $\mathrm{Fe}^{3+}$ (VI).

Recent work on transilion metal hydridocomplexes strongly suggests that hydrogen

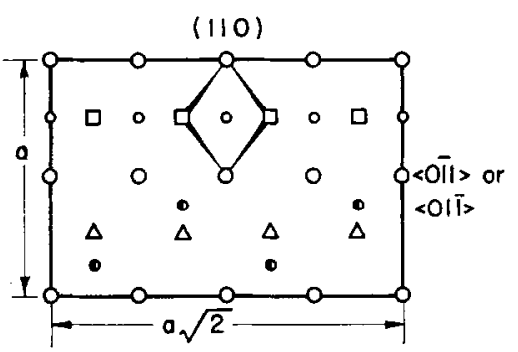

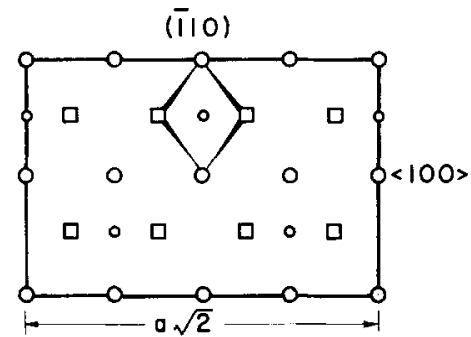

O OXYGEN

- cOBALT (VI), $\mathrm{Co}^{+2}, \mathrm{Co}^{+3}$

- IRON (VI), $\mathrm{Fe}^{+2}, \mathrm{I} / 2 \mathrm{Fe}^{+3}$

- IRON (IV), $1 / 2 \mathrm{Fe}^{+3}$ (a/8 below plane)

a Adsorption site (VI), obove plane

$\triangle$ Adsorption site (IV), above plane

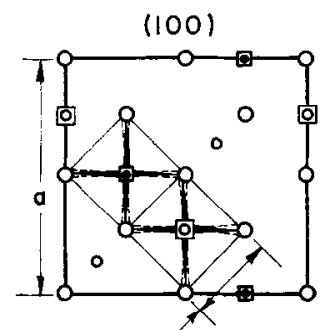

$\frac{a \sqrt{2}}{4}$

FIG. 12. Composition of (100), (110), and (110) planes in $\mathrm{CoFe}_{2} \mathrm{O}_{4}$.

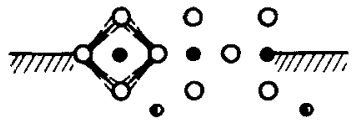

(a)

ORIGINAL SURFACE

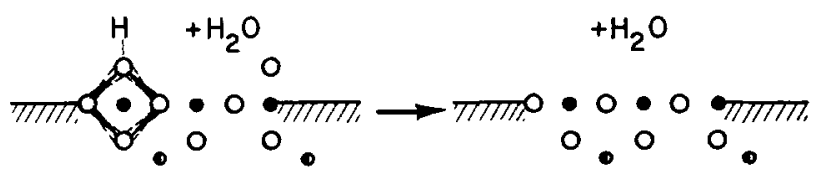

(b) (c)

HYDROGEN ACTIVATION

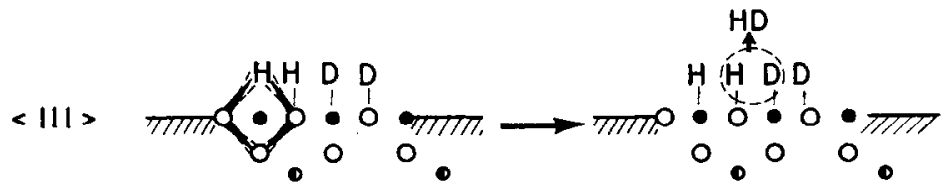

(d)

(e)

HYDROGEN ADSORPTION

HD FORMATION

O OXYGEN

- METAL (VI)

Surface plane (OII)

- metal (IV)

Section plane (100)

Fif, 13. Schematic representation of surface changes in the (100) plane during activation, adsorption of hydrogen, and hydrogen-deuterium exchange. 
is incorporated in the complexes as an anionic ligand (13). The condition for the presence of this type of bonding is the presence of ligands around the metal with high ligand field strength $\left(\mathrm{Cl}^{-}, \mathrm{CN}^{-}\right)$. In the present context the oxygen anions could form these ligands and produce, together with the hydrogen, octahedral surface complexes in which the metal ion retains its normal valency and coordination number. From studies on hydrido-complexes it is also known that the hydrogen bonding is very sensitive to the environment, i.e., to the nature of the remaining ligands. Assuming that the analogy between hydrogen chemisorption on spinels and bonding in hydrido-complexes of metals is valid, it is possible to use the latter effect to interpret the critical importance of surface pretreatment to the reactivity of the surface itself.

In order to obtain an insight into the controlling step of the exchange reaction, it is necessary to arrive at an estimate of the fractional surface coverage at reaction conditions. The results from the thermoelectric power measurements can provide some guide to this purpose. In previous studies on thermoelectric power of particulate systems $(8)$, it was calculated that the variation in thermoelectric power with the amount of adsorbate could be expressed by the equations:

$$
\begin{aligned}
& \frac{\left(n_{1}\right)_{\mathrm{c}}}{\left(n_{1}\right)_{0}}=\exp \left[-\frac{\omega}{\delta} \frac{\left(Q^{\mathrm{c}}-Q^{0}\right)}{k}\right], \text { for } \delta<\omega \\
& \frac{\left(n_{1}\right)_{\mathrm{c}}}{\left(n_{1}\right)_{0}}=\exp \left\{\frac{Q^{\mathrm{c}}-Q^{0}}{k}\right\}, \text { for } \delta \geqslant \omega
\end{aligned}
$$

A value for $\delta=\left(\epsilon R T / 8 \pi n e^{2}\right)^{1 / 2}$ can be computed for a typical case. Thus, in $\mathrm{Co}_{0.92-}$ $\mathrm{Fe}_{2.07} \mathrm{O}_{4}$ with $3.15 \times 10^{20} \mathrm{Fe}^{2+} \mathrm{cm}^{-3}, T=$ $150^{\circ} \mathrm{C}$, and taking $\epsilon \approx 12$, one obtains $\delta \cong 2 \dot{\mathrm{A}}$. This shows that it is justifiable to assume that during the catalytic experiments no appreciable electrical potential difference was set up at the surface. Under these conditions, it is likely that Eq. (6) applics. Since the value of $\omega$ is not known, we shall use Eq. (7) for calculation, and the result will give an upper limiting value for $\left(n_{1}\right)_{e}$. Using the values from Fig. 6 [single carrier conductor, Eq. (3) applies], $Q^{0}=-470$ $\mu$ volt ${ }^{\circ} \mathrm{C}^{-1}$ and $Q^{\mathrm{c}}=-385 \mu$ volt ${ }^{\circ} \mathrm{C}^{-1}$, one obtains $\left(n_{1}\right)_{\mathrm{c}} /\left(n_{1}\right)_{0}=2.66$. From Eq. (3) and the fact that the sample initially contained 0.04 conduction electron molecule ${ }^{-1}$, $\left(n_{1}\right)_{0}=1.17 \times 10^{20} \mathrm{~cm}^{-3}$. Then $\left(n_{1}\right)_{\mathrm{c}}=2.66$ $\times 1.17 \times 10^{20}=3.1 \times 10^{20} \mathrm{~cm}^{-3}$. The per cent of surface iron sites which have been occupied by hydrogen adsorption is $[(3.1 \times$ $\left.\left.10^{20}\right)-\left(1.17 \times 10^{20}\right) / 0.45 \times 10^{22}\right] \times 100=$ $4 \%$, where $0.45 \times 10^{22} \mathrm{~cm}^{-3}$ is the concentration of $\mathrm{Fe}^{3+}$ ions in octahedral sites. This calculation assumes that on samples with $x>2$ no hydrogen is adsorbed on $\mathrm{Co}^{2+}$ and $\mathrm{O}^{2-}$ ions, and only $\mathrm{Fe}^{3+}(\mathrm{VI})$ ions are chemisorption sites. These assumptions are likely to produce errors in opposite directions on the calculated value of the fractional coverage. Therefore, it is believed that a surface coverage of a few per cent is a reasonable value. It should be noted that this order of magnitude is consistent with the calculation reported in the previous section on the amount of adsorbed hydrogen needed to swing the thermoelectric power from the positive to the negative maxima of samples with $x>2$.

Let us assume that following heterolytic adsorption on the spinel surface hydrogen does not easily migrate among surface complexes (immobile adsorption) and that the rate of the exchange is controlled by desorption (Fig. 13e); then the expression of the reaction rate calculated by absolute rate theory is (5):

$$
\exp (E / R T)\left(v_{\text {des }}\right)_{\text {imm }}=2 L \theta^{2}(k T / h)\left(F^{\dagger} / F_{\text {a }}\right)
$$

where the ratio $F \dagger / F_{\mathrm{a}} \approx 1$, since activated complex and adsorbed species are assumed to be immobile. Taking $T=100^{\circ} \mathrm{C}, \theta=0.2$. $L=6.9 \times 10^{14}$ atoms $\mathrm{cm}^{-2}$, one gets

$$
\begin{aligned}
& \exp \left(\boldsymbol{E} / R^{\prime} T^{\prime}\right)\left(v_{\mathrm{des}}\right)_{\mathrm{imm}}= \\
& 4.38 \times 10^{24} \text { molecules } \mathrm{cm}^{-2} \mathrm{sec}^{-1}
\end{aligned}
$$

From the results of the experimental runs at low temperature (high catalytic activity), it was calculated $\ln \left(k_{0}\right)=30.1$ and $E=18.3$ kcal mole ${ }^{-1}$ for samples with $x=1.93$ (Table 2). At $100^{\circ} \mathrm{C}$, these data give a first order rate constant, $k=2.064 \times 10^{2} \mathrm{~min}^{1}$ and $p=2.82 \times 10^{-2}$. Using the following values: $\varphi=0.43 \mathrm{~cm}^{3}, D_{2}=0.02, v_{\mathrm{s}}=20$ 
$\mathrm{cm}^{3} \min ^{-1}, T_{\mathrm{a}}=26^{\circ} \mathrm{C}, P_{0}=740 \mathrm{~mm} \mathrm{Hg}$, $w=4.12 \mathrm{~g}$, and $A=8 \times 10^{-2} \mathrm{~m}^{2} \mathrm{~g}^{-1}$, the experimental rate of exchange at $100^{\circ} \mathrm{C}$ is:

$$
\begin{aligned}
\exp \left(\frac{E}{R T}\right) v_{\exp }=\frac{D_{2} p N_{\mathrm{A}}}{A u \tau}=\frac{D_{2} p N_{\mathrm{A}} v_{\mathrm{a}} P_{\mathrm{a}}}{R T_{\mathrm{a}} A w} \\
=8.02 \times 10^{24} \text { molecules } \mathrm{cm}^{-2} \mathrm{sec}^{-1}
\end{aligned}
$$

This value is within an order of magnitude of the theoretical one. Theoretical rates have also been calculated on the basis of other possible slow reaction steps: mobile and immobile adsorption, mobile dissociative desorption, first order reaction. In none of these instances was there agreement between calculated and experimental rates. This lends support to an exchange mechanism controlled by desorption kineties.

According to the reaction scheme suggested the catalytic exchange is dependent upon the stability of surface complexes of the type $\mathrm{Me}^{3+}\left(\mathrm{OH}^{-}\right)\left(\mathrm{H}^{-}\right)\left(\mathrm{O}^{2-}\right)_{4}$. In cobalt ferrite the $\mathrm{Co}^{3+}$ ions are situated about 0.5 ev below the $\mathrm{Fe}^{3+}$ ions (4), but the relative stability of the surface hydrido-complexes is not known. The crucial factor in the stabilization is the energy separation between bonding and antibonding levels, the extent of the gap varying according to whether the complexes are formed with $\mathrm{Co}^{2+}, \mathrm{Co}^{3+}, \mathrm{Fe}^{2+}$, or $\mathrm{Fe}^{3+}$ ions. As a crude approximation one may look at the relative stability of bulk metal hydrides for clues on the stability of the surface metal hydrides. In the former compounds it is well known that, upon moving from left to right across the pcriodic table of the elements, the bonding of transition metal hydrides becomes less ionic with a decrease in the heat of formation. A similar criterion applied to the surface hydride would indicate that the relative stability of the complexes should follow $\mathrm{Fe}^{3+}\left(\mathrm{H}^{-}\right)(\mathrm{OH})^{-}\left(\mathrm{O}^{2-}\right)_{4}>\mathrm{Co}^{3+}\left(\mathrm{H}^{-}\right)-$ $\left(\mathrm{OH}^{-}\right)\left(\mathrm{O}^{2-}\right)_{4}$. Then, if the decomposition of the complexes is the slow step for the exchange reaction, the activation energies corresponding to iron and cobalt sites should be in the sequence $E_{\mathrm{Fe}}>E_{\mathrm{Co}}$, as has been found experimentally (Table 2).

Oxygen poisoning of the activated surface can be explained by postulating a preferential adsorption of oxygen atoms on surface complexes in such a manner as to re-estab- lish the sixfold coordination around $\mathrm{Co}^{3+}$ and $\mathrm{Fe}^{3+}$.

The picture which emerges from the previous considerations on the role of $\mathrm{Co}^{3+}$ and $\mathrm{Fe}^{3+}$ ions in the catalytic exchange is in accord with previous claims on the formation of preferential catalytic sites on trivalent cations of spinel structures (5).

Finally, it is pertinent to emphasize the fact that surface space charge effects are not likely to be a contributing factor to the surface activity of the catalysts investigated, since hydrogen adsorption on $n$-type samples is not expected to introduce a spacecharge layer and the behavior of these catalysts is not much different from the behavior of $p$-type catalysts. Possibly this results from the rather thin space-charge layer formed on $p$-type samples.

\section{Conclusions}

Cobalt ferrite catalysts can be advantageously used to analyze the role of electron defects on the rate of the hydrogen-deuterium exchange reaction. Under conditions in which the ferrite is a single carricr scmiconductor, no significant difference in the rate of the reaction, at temperatures from $60^{\circ}$ to $110^{\circ} \mathrm{C}$, is found between $p$-and $n$-type samples. Since the investigation was performed on ferrite surfaces with high catalytic; activity, this result shows that, under the experimental conditions used in this study, the nature and concentration of the electrical carrier play no kinetically decisive role in the catalytic exchange. The possibility of the formation of surface complexes not entailing a change in the oxidation state of the metal ion seems to be a more important factor for the adsorption and desorption of hydrogen. The application of absolute rate theory indicates that hydrogen desorption is the kinetically controlling step. This conclusion is consistent with a qualitative explanation of the differences in activation energies of the exchange reaction among catalysts with different values of $x$, based on the relative stability of the surface metalhydrogen complexes.

\section{Srmbols}

$8.39 \mathrm{~A}^{\circ}$, lattice parameter of $\mathrm{CoFe}_{2} \mathrm{O}_{4}$ Surface area $\left[\mathrm{m}^{2} \mathrm{~g}^{-1}\right]$ 
$c_{\mathrm{a}} \quad$ Number of occupied surface sites

$c_{\mathrm{g}} \quad P N_{\mathrm{A}} / R T$, number of molecules in gas phase [molecules $\mathrm{cm}^{-3}$ ] at the experimental temperature and pressure

$e \quad$ Electron charge

$E \quad$ Activation energy [kcal mole ${ }^{-1}$ ]

$E_{\mathrm{f}} \quad$ Energy of Fermi level [ev]

$E_{\mathrm{g}} \quad$ Width of energy gap [ev]

$F_{\mathrm{a}} \quad$ Partition function of adsorbed species

$F \dagger \quad$ Partition function of activated complex

$h \quad$ Planck constant

$k \quad$ Boltzman constant, first order reaction rate constant $\left[\mathrm{min}^{-1}\right]$

$k_{0} \quad$ Pre-exponential factor in first order rate equation $\left[\mathrm{min}^{-1}\right]$

$L \quad c_{\mathrm{s}}+c_{\mathrm{a}}$, total number of surface sites [atoms $\mathrm{cm}^{-2}$ ]

$n \quad$ Concentration of electrons $\left[\mathrm{cm}^{-3}\right]$ (subscript 1) or electron holes (subscript 2), subscripts c and 0 for chemisorption or before chemisorption, respectively

$N \quad 1.35 \times 10^{22}\left[\mathrm{~cm}^{-3}\right]$, concentration of octahedral cations in $\mathrm{CoFe}_{2} \mathrm{O}_{4}$

$N_{\mathrm{A}} \quad$ Avogadro Number

$p \quad \frac{\left[\mathrm{HD} / \mathrm{H}_{2}\right]_{t}}{\left[\mathrm{HD} / \mathrm{H}_{2}\right]_{t}+\left[\mathrm{D}_{2} / \mathrm{H}_{2}\right]_{t}} \times 100=$ $\frac{[\mathrm{HD}]_{t}}{[\mathrm{HD}]_{t}+2\left[\mathrm{D}_{2}\right]_{t}} \times 100$, \% of conversion

$P$ Pressure (subscript a refers to ambient conditions)
Q Thermoelectric power $\left[\mu\right.$ volts $\left.{ }^{\circ} \mathrm{C}^{-1}\right]$ (superscripts $c$ and 0 refer to the value obtained upon chemisorption and before chemisorption, respectively)

$R \quad$ Gas constant

$T$ Temperature (subscript a refers to ambient conditions)

$v$ Rate of reaction [molecules $\mathrm{cm}^{-2}$ sec $^{-1}$ ]

flow rate $\left[\mathrm{cm}^{3} \mathrm{~min}^{-1}\right]$

$w \quad$ catalyst weight [g]

$\alpha, \beta \quad$ energy terms for electron and electron holes transport, respectively (4)

$\delta \quad$ Debye length

e Dielectric constant

$\theta \quad c_{\mathrm{a}} / L$, fraction of surface covered with adatoms

$\mu \quad$ Mobility of electrons (subscript 1) or electron holes (subscript 2); micron

Contact time [min]

$\varphi \quad$ Void volume in reactor $\left[\mathrm{cm}^{3}\right]$

$\omega \quad$ Thermal gradient

(IV) Refers to metal ions with fourfold coordination in the spinel structure

(VI) Refers to metal ions with sixfold coordination in the spinel structure

\section{REFERENCES}

1. Kuchaev, J. L., and Boneskov, G. K., Problemy Kinetiki $i$ Kataliza Akad. Nauk. S.S.S.R. 10, 108 (1960).

2. Simkovich, G., And Wagner, C., J. Catalysis I, 521 (1962).

\section{APPENDIX I}

Characteristics of Some Crystal Planes in $\mathrm{CoF}_{2} \mathrm{O}_{4}$

\begin{tabular}{|c|c|c|c|c|c|c|}
\hline Plane & Area & $\begin{array}{l}\text { Composition } \\
\text { (atoms area }{ }^{-1} \text { ) }\end{array}$ & $\begin{array}{l}\text { Atomic density } \\
\text { (atoms } a^{-2} \text { ) }\end{array}$ & $\begin{array}{l}\text { No. of } \\
\text { bonds }\end{array}$ & $\begin{array}{l}\text { No. of } \\
\text { adsorption sites }\end{array}$ & Sites/cation \\
\hline$\{100\}$ & $a^{2}$ & $\begin{array}{l}80 \\
2 \mathrm{Co}(\mathrm{VI}) \\
2 \mathrm{Fe}(\mathrm{VI})\end{array}$ & 12 & 12 & $4(\mathrm{VI})$ & 1 \\
\hline (110) & $a^{2} \sqrt{2}$ & $\begin{array}{l}8 \mathrm{O} \\
4 \mathrm{Co}(\mathrm{VI}) \\
4 \mathrm{Fe}(\mathrm{IV})\end{array}$ & 11.3 & 18 & $\begin{array}{l}4(\mathrm{VI}) \\
4(\mathrm{IV})\end{array}$ & 1 \\
\hline$(\overline{1} 10)$ & $a^{2} \sqrt{2}$ & $\begin{array}{l}8 \mathrm{O} \\
4 \mathrm{Co}(\mathrm{VI})\end{array}$ & 7.5 & 18 & $8(\mathrm{VI})$ & 2 \\
\hline$(\mathbf{1} \overline{1} \overline{1})$ & $a^{2} \sqrt{3}$ & $\begin{array}{l}2 \mathrm{Co}(\mathrm{VI}) \\
4 \mathrm{Fe}(\mathrm{VI})\end{array}$ & 3.5 & 18 & $10(\mathrm{VI})$ & 1.66 \\
\hline
\end{tabular}

a Assumed to be equal to the number of atoms necessary to restore the typical coordination of underlying cations. 
3. Kobayashi, H., and Wagner, C., J. Chem. Phys. 26, 1609 (1957).

4. Jonker, G. H., J. Phys. Chem. Solids 9, 165 (1959).

5. Schwab, G. M., Roth, E., Grintzos, C., and Mavrakis, N., in "Structure and Properties of Solid Surfaces," p. 464. Univ. of Chicago Press, Chicago, Illinois, 1953.

6. Squires, R. G., Ph.D. Thesis, University of Michigan, 1962.

\%. Smiltens, R., J. Am. Chem. Soc. 79, 4881 (1957) ; Robin, J., and Benard, J., Compt. rend. 232, 1830 (1951); Compt. rend. 23, 734 (1952); Roiter, B. D., and Paladino, A. E., J. Am. Ceram. Soc. 45, 128 (1962).

8. Parravano, G., and Domenicali, C. A., $J$. Chem. Phys. 26, 359 (1957).

9. Kubokawa, Y., and Tayama, O., J. Phys. Chem. 60, 823 (1956); Eischens, R. P.,
Pliskin, W. A., and Law, M. J. D., J. Catalysis 1, 180 (1962).

10. Gray, T. J., and Darby, P. W., J. Phys. Chem. 60, 201 (1956) ; Brelanski, A., Deren, J., Haber, J., Sloczynski, J., and Wilkowa, 'I', Bull. Acad. Polon. Sci. 7, 5 (1959).

11. Stone, F. S., Adv. in Catalysis, 13, 1 (1962).

12. Dowdes, D. A., ANd Wells, D., in "Actes du Deuxieme Congres International de Catalyse," p. 1499. Editions Technip, Paris, 1961.

13. Chatт, J., Proc. Chem. Soc. p. 318 (1962); J. Chem. Soc., p. 5075 (1962).

14. Molinari, E., and Parravano, G., J. Am. Chem. Soc. 75, 5233 (1953).

15. Scholten, J. F., Zwietering, P., Konvalin Ka, A., and DeBoer, J. H., Trans. Faraday Soc. 55, 2166 (1959). 